\title{
Autonomous process fault diagnosis and root cause analysis for processing system using one class SVM and NN permutation algorithm
}

\author{
Rajeevan Arunthavanathan, Faisal Khan*, Salim Ahmed, Syed Imtiaz
}

Centre for Risk, Integrity, and Safety Engineering (C-RISE), Faculty of Engineering and Applied

Science, Memorial University of Newfoundland, St. John's NL, Canada A1B 3X5

*Correspondence author: fikhan@mun.ca

Table S1. TE process continues process variables.

\begin{tabular}{|c|c|c|c|}
\hline Index & Description & Index & Description \\
\hline XMEAS1 & A feed (stream 1) & XMEAS12 & Separator level \\
\hline XMEAS2 & D feed (stream 2) & XMEAS13 & Separator pressure \\
\hline XMEAS3 & E feed (stream 3) & XMEAS14 & Separator underflow (stream 10) \\
\hline XMEAS4 & Total feed (stream 4) & XMEAS15 & Stripper level \\
\hline XMEAS5 & Recycle flow (stream 8) & XMEAS16 & Stripper pressure \\
\hline XMEAS6 & Reactor feed rate (stream 6) & XMEAS17 & Stripper underflow (stream 11) \\
\hline XMEAS7 & Reactor pressure & XMEAS18 & Stripper temperature \\
\hline XMEAS8 & Reactor level & XMEAS19 & Stripper stream flow \\
\hline XMEAS9 & Reactor temperature & XMEAS20 & Compressor work \\
\hline XMEAS10 & Purge rate (stream 9) & XMEAS21 & Reactor cooling water outlet temperature \\
\hline XMEAS 11 & Separator temperature & XMEAS22 & $\begin{array}{l}\text { Condenser cooling water outlet } \\
\text { temperature D }\end{array}$ \\
\hline
\end{tabular}


Table S2. Selected TE process fault condition for testing.

\begin{tabular}{|l|l|l|}
\hline Fault ID & Description Variable & Type \\
\hline IDV1 & A/C feed ratio, B composition constant (Stream 4) & Step \\
IDV4 & Reactor cooling water inlet temperature & Step \\
IDV5 & Condenser cooling water inlet temperature & Step \\
IDV6 & A feed loss (Stream 1) & Step \\
IDV11 & Reactor cooling water inlet temperature & Random variation \\
IDV12 & Condenser cooling water inlet temperature & Random variation \\
IDV14 & Reactor cooling water valve & Sticking \\
IDV15 & Condenser cooling water valve & Sticking \\
\hline
\end{tabular}

